\title{
EPISTEMOLOGÍA CRAIGUIANA Y EL ROL DE LAS INTUICIONES EN EL TEORIZAR EPISTEMOLÓGICO*
}

Leandro De Brasi**

ldebrasi@uahurtado.cl

RESUMEN El uso de las intuiciones como evidencia es ubicuo en la epistemología, pero no poco controvertido. Por más de una década y media, algunos epistemólogos experimentales han cuestionado esta apelación a las intuiciones epistémicas. Estos filósofos explotan los métodos de la psicología experimental, en particular métodos de encuestas, para poner a prueba cuán ampliamente compartidas son algunas intuiciones. Dado su compromiso con la relevancia de la investigación empírica a la construcción de teorías filosóficas, estos filósofos califican como naturalistas metodológicos. Pero algunos naturalistas metodológicos que también recomiendan renunciar a esa apelación de la intuición como evidencia no basan su caso en encuestas. En este artículo considero dos desafíos fundamentales a la práctica estándar de apelar a las intuiciones que estos filósofos naturalistas plantean y argumento que un proyecto epistemológico inspirado en el trabajo de Edward Craig le permite, en principio, al epistemólogo que comparte ese naturalismo metodológico lidiar con ellos. De todas formas concluyo que, independientemente del éxito del enfoque Craiguiano, las intuiciones tienen un rol que jugar en el teorizar epistemológico en relación a la adjudicación entre enfoques.

Palabras claves Edward Craig, Epistemología Experimental, Naturalismo Metodológico, Clase Social.

* El presente trabajo fue realizado dentro del marco del proyecto Fondecyt \#11140279 (Chile). Artigo submetido em 20/02/17. Aceito em 21/05/17.

** Alberto Hurtado University. Santiago - Chile.

KRITERION, Belo Horizonte, no 140, Ago./2018, p. 533-552 
ABSTRACT The use of intuitions as evidence is widespread in epistemology, but not uncontroversial. For over a decade and a half, some experimental epistemologists have called into question this appeal to epistemic intuitions. These philosophers bring the methods of experimental psychology, in particular survey methods, to bear on epistemological theorizing. Given their commitment to the relevance of empirical work to philosophical theory construction, they qualify as methodological naturalists. But some methodological naturalists who also recommend giving up appeals to intuition as evidence do not base their case on experimental surveys. In this article I consider two main challenges to the standard practice of appealing to intuitions posed by these naturalist philosophers and argue that a Craig-style epistemological project can allow the methodological naturalist to address them. Anyway I conclude that, regardless of the truth of the Craigian approach, intuitions still have a role to play in epistemological theorizing in the adjudication between approaches.

Keywords Edward Craig, Experimental Epistemology, Methodological Naturalism, Social Kind.

\section{Introducción}

El uso de intuiciones como evidencia es ubicuo en la epistemología (y en la filosofía en general ${ }^{1}$ ), pero no poco polémico. ${ }^{2}$ Por más de una década y media,

1 A través de la historia de la filosofía, las tesis filosóficas han sido apoyadas, modificadas y rechazadas en base a ejemplos y contra-ejemplos intuitivos. Considere, por ejemplo, el método Socrático de investigación (elenchus), en donde una definición propuesta por un interlocutor es rechazada si está sujeta a un contraejemplo (dado que la definición debiera estar exenta de excepciones). Este resultado contra-intuitivo le permite al personaje "Sócrates" rechazar la presunta definición porque excluye casos legítimos o incluye ilegítimos. Por ejemplo, cuando Céfalo afirma que la justicia es decir la verdad y devolver lo prestado, Sócrates nota que devolverle un arma a un amigo que ha enloquecido no es justo y tanto Sócrates como Céfalo toman ese contra-ejemplo como una refutación de la definición propuesta, dado que permite que acciones intuitivamente injustas sean clasificadas como justas ("República" 331c-d). Otra estrategia Socrática para argumentar en contra de una afirmación es la de exponer inconsistencias por medio de pequeños reductios ad absurdum. La razón para concentrarse en pequeños reductios, en lugar de grandes, es dialéctica. Pequeños reductios tienen mayor fuerza dialéctica, pero es probable también que hagan uso de intuiciones (considere, por ejemplo, el caso de Sócrates contra el relativismo de Protágoras en "Teeteto" 157d y sig.). Estas, por supuesto, no son las únicas estrategias Socráticas. Hay otras estrategias que no conciernen intuiciones, como ser: encontrar ambigüedades en las definiciones de términos (considere, por ejemplo, el caso de Sócrates contra la segunda definición de piedad en "Eutifrón" 7a).

2 De hecho, este uso ubicuo de las intuiciones como evidencia ha sido reconocido tanto como por amigos como enemigos de las intuiciones-excepciones notables son Cappelen (2012), Deutsch (2015). Por ejemplo, George Bealer, un amigo de las intuiciones, afirma que las intuiciones en la filosofía son tratadas como evidencia y por lo tanto forman parte del "procedimiento justificativo estándar" que los filósofos utilizan para apoyar sus creencias y teorías (1998, p. 204). Y Jaakko Hintikka, un enemigo de (el uso actual de) las intuiciones, asevera que "uno de los métodos argumentativos favoritos de los filósofos contemporáneos es apelar a las intuiciones" 
algunos epistemólogos experimentales han cuestionado esta apelación a las intuiciones epistémicas (Weinberg et al., 2001). De hecho, algunos afirman que "la evidencia experimental sugiere lo inadecuadas que son las intuiciones para servir como evidencia" (Alexander y Weinberg, 2007, p. 63; mi traducción). Estos filósofos explotan los métodos de la psicología experimental, en particular métodos de encuestas, para poner a prueba cuán ampliamente compartidas son algunas intuiciones. Dado su compromiso con la relevancia de la investigación empírica a la construcción de teorías filosóficas, estos filósofos califican como naturalistas metodológicos. Pero algunos naturalistas metodológicos que también recomiendan renunciar a la apelación de la intuición como evidencia no basan su caso en encuestas (Kornblith, 2006).

En este artículo considero dos desafíos fundamentales a la práctica estándar de apelar a intuiciones que estos filósofos naturalistas plantean y argumento que un proyecto epistemológico inspirado en el trabajo de Edward Craig le permite al epistemólogo que comparte ese naturalismo metodológico lidiar con ellos. ${ }^{3}$ Por lo tanto, dado tal proyecto, uno no debería, como naturalista metodológico, considerar tales desafíos como problemáticos. Los dos desafíos que nos concernirán aquí son los siguientes.

El primer desafío consiste en acomodar los resultados de la epistemología experimental "negativa." Esta investigación empírica sugiere que las intuiciones epistémicas varían de acuerdo a factores epistémicamente irrelevantes. Por ejemplo, intuiciones acerca de casos Gettier (Gettier, 1963) parecen exhibir variaciones interculturales (Weinberg et al., 2001) e intuiciones acerca de casos Truetemp (Lehrer, 1990) parecen ser susceptibles a efectos de orden (Swain et $a l ., 2008)$. Esto parece crear dudas sobre la relevancia de las intuiciones a la investigación epistemológica a menos que podamos discernir cuáles privilegiar para construir nuestras teorías. El segundo desafío introduce la posibilidad de que nuestras intuiciones acerca de los fenómenos extra-mentales a ser explicados no son una guía correcta acerca de las verdaderas naturalezas de esos fenómenos dado que nuestros conceptos están infectados por ignorancia y error (Kornblith, 2006, 2007). Nuestras intuiciones pueden, como mucho, revelar nuestros conceptos, pero los conceptos no son siempre los objetos de nuestro teorizar epistemológico. Esta potencial brecha entre los conceptos y

(1999, p. 127; mi traducción). De hecho, las intuiciones no solo son normalmente asignadas un rol evidencial en la epistemología y, mas generalmente, en la filosofía, sino también un rol epistémico privilegiado-ver e.g. Kripke (1980, p. 42).

3 Más específicamente, aquí nos concentraremos en su libro "Knowledge and the State of Nature" (1990). 
los fenómenos a estudiar parece sugerir que las intuiciones no deberían jugar algún tipo de rol en la investigación epistemológica.

El artículo prosigue de la siguiente manera. Después de algunas cuestiones preliminares ( $(2)$, considero los dos desafíos anteriormente mencionados bajo la luz de un enfoque Craiguiano ( $\S 3-4)$. A continuación, ofrezco algunas observaciones a modo de conclusión que sugieren que las intuiciones todavía poseen un rol que desempeñar en el teorizar epistemológico independientemente del éxito de tal enfoque (\$5).

\section{Intuiciones, evidencia y naturalismo}

Dados nuestros intereses, tres preguntas centrales surgen. Sus respuestas nos ayudarán a crear un trasfondo en común para considerar si los desafíos anteriormente mencionados son exitosos. Estas preguntas son: (a) ¿Qué son las intuiciones? (b) ¿Qué es la evidencia? (c) ¿Qué es el naturalismo metodológico? Considerémoslas en ese orden.

(a) No hay una concepción de intuiciones consensuada, pero aquí tomo a las intuiciones como juicios no-inferenciales que surgen espontáneamente, pero no necesariamente instantáneamente, sin una fenomenología distintiva. ${ }^{4}$ Juicios intuitivos son estados conscientes que poseen contenido proposicional y que no deben ser confundidos con presentimientos, sentimientos o impresiones. Además, como las intuiciones son juicios directos, no-inferenciales, no son el resultado de un razonamiento inferencial consciente. ${ }^{5}$ También (y en aras del argumento, dado el segundo desafío anteriormente mencionado) asumo que los conceptos son causalmente responsables por estos juicios intuitivos y que los conceptos son entidades psicológicas, pero permaneceré neutral acerca de otros aspectos (controvertidos) de su naturaleza.

Por otra parte, deberíamos también diferenciar entre dos tipos de intuiciones: intuiciones particulares y generales. Las intuiciones particulares identifican un caso particular como un F o un no-F (por ejemplo, si una instancia particular es un caso de conocimiento). Las intuiciones generales identifican una relación entre distintas propiedades, $\mathrm{F}$ y $\mathrm{G}$ (por ejemplo, la intuición acerca de la relación entre ser conocimiento y ser verdadero) y la relación entre distintos particulares que son F (por ejemplo, la intuición sobre el principio de clausura: aproximadamente,

4 Ver también Mortensen y Nagel (2016); cf. Chudnoff (2013). Otras concepciones son ofrecidas en e.g. Booth y Rowbottom (2014); ver Alexander (2012), para un útil resumen de posiciones.

5 Ver e.g. las "Reglas para la dirección del espíritu" (3) y "Principios de filosofía" (45-6) de Descartes. 
si sé que $p$ y sé que $p$ implica $q$, sé que $\left.q^{6}\right)$. Para ilustrar la diferencia, considere la siguiente "paradoja escéptica" (Cohen, 1988):

A. Sé que tengo una mano

B. No sé que no soy un cerebro en una cubeta

C. Si sé que tengo una mano, sé que no soy un cerebro en una cubeta

En este caso, tenemos (supuestamente) tres afirmaciones individualmente plausibles que parecen conjuntamente inconsistentes, ${ }^{7}$ en donde A y B poseen una cierta plausibilidad porque están respaldadas por intuiciones particulares acerca de instancias de conocimiento (o falta de conocimiento) y $\mathrm{C}$ porque deriva del intuitivo (y no restringido) principio de clausura, dado el conocimiento de la implicancia. ${ }^{8}$

Ahora bien, experimentos mentales en la epistemología normalmente conciernen intuiciones acerca de casos particulares (i.e. intuiciones particulares), en vez de intuiciones generales. ${ }^{9}$ De hecho, algunos filósofos contemporáneos (abiertamente) privilegian intuiciones particulares por sobre las generales (e.g. Chisholm, 1982; Greco, 2000). Sin embargo, este "particularismo" acerca de las intuiciones (i.e. la posición que solo las intuiciones particulares poseen peso evidencial o poseen mayor peso evidencial que las intuiciones generales) no es el único enfoque disponible, ni mucho menos adoptado.

En efecto, concerniente a los datos para construir teorías del conocimiento, Roderick Chisholm (1982) distingue entre el generalismo (o metodismo) y el particularismo. ${ }^{10}$ Según Chisholm, algunos filósofos son particularistas ya que ponen a prueba sus teorías por medio de creencias acerca de casos particulares, para las cuales las intuiciones particulares pueden proveer evidencia. Otros

6 Refinamientos a esta formulación del principio parecen necesarios; ver e.g. Hawthorne (2004; 2014). Sin embargo, como Baumann (2011, p. 599) afirma, la formulación ofrecida encierra la idea central detrás del principio de clausura (cf. Dretske, 2010, p. 133). De todas formas, como enemigos del principio también admiten (e.g. Nozick, 1981), un principio de clausura así o similarmente formulado es muy intuitivo. Para más detalles sobre la intuición detrás del principio, ver De Brasi (2014).

7 Aquí ignoro la cuarta intuición sobre la relación entre esas tres proposiciones intuidas: i.e. la intuición que A, B y C son conjuntamente inconsistente (cf. Conee, 2014). Para más detalles sobre este tipo de intuiciones, ver Pollock (1974).

8 Para un argumento a favor de la restricción del principio, ver De Brasi (2014).

9 Experimentos mentales (usualmente acerca de casos imaginarios y a veces un tanto bizarros) son elaborados como "bombines de intuiciones" (intuition pumps como dice Daniel Dennett). Note que la intención de Dennett (1980), al acuñar la frase, era derogatoria, ya que cree que el experimento mental del cuarto chino (Chinese room) de John Searle no constituye para nada un argumento-ver también Dennett (1987). Esto, sin embargo, no significa que Dennett no utilice intuiciones de vez en cuando-ver e.g. Dennett (2013, en particular §XI). Esto último no intenta ser un simple argumento ad hominem; después de todo, las dos posiciones son compatibles. De hecho, ver Dennett y Hofstader (1982, p. 230), en donde afirman que experimentos mentales demasiado extravagantes podrían no ser fiables. La idea de esa afirmación es mostrar que, más allá de la legitimidad de la discriminación propuesta (aunque esta no parece ser descabellada-ver nota 12), uno no debe asumir que todas las intuiciones son utilizadas como evidencia en la práctica justificativa estándar (ver nota 2).

10 Ver también Sosa (1991, pp. 158, 165-7). 
filósofos son generalistas ya que ponen a prueba sus teorías por medio de creencias acerca de criterios y principios, para las cuales las intuiciones generales pueden proveer evidencia. Estos dos enfoques metodológicos (positivos) son el resultado de lo que Chisholm toma como las dos preguntas centrales al respecto:

i. “¿Qué sabemos? ¿Cuál es la extensión de nuestro conocimiento?”

ii. “¿Cómo vamos a decidir si sabemos? ¿Cuáles son los criterios relacionados al conocimiento?" (1982, p. 65; mi traducción)

Por lo tanto, si uno sabe la respuesta a una de estas dos preguntas, uno puede idear una respuesta a la otra. El particularista cree que posee la respuesta a (i) e intenta de esa manera responder a (ii); mientras que el generalista cree poseer la respuesta a (ii) y así responder a (i).

Sin embargo, no es claro que haya generalistas o particularistas puros. Después de todo, tanto intuiciones generales como particulares se encuentran normalmente en conflicto para un individuo dado en un momento dado y dichos conflictos se toman en cuenta seriamente. Esto, por ejemplo, se aprecia claramente en la paradoja escéptica anterior. Las intuiciones particulares (A y B) entran en conflicto solo porque aceptamos el intuitivo principio de clausura. Si uno fuese un particularista puro, uno debería aceptar las dos intuiciones particulares y, dado que la intuición general crea el conflicto entre ellas, rechazarla (i.e. rechazar la intuición sobre el principio de clausura). Eso es, un particularista puro no se preocuparía por tal conflicto de intuiciones. Pero epistemólogos parecen tomar en cuenta (aunque de distintas maneras) ambas baterías de intuiciones. De hecho, lo que muchos epistemólogos parecen hacer es capturar lo que toman como las intuiciones generales y particulares más fuertes: eso es, intentan alcanzar un equilibrio reflexivo (estrecho). ${ }^{11} \mathrm{Y}$, en línea con esta práctica común, no excluiremos a ninguno de estos dos tipos de intuiciones y lo que sigue aplica a ambos tipos aun cuando la atención, en la literatura (e inclusive en este artículo), está generalmente puesta en las intuiciones particulares.

(b) Como en el caso anterior, no hay una noción de evidencia consensuada (ver e.g. Kelly, 2008). Aquí, sin embargo, tomaremos $x$ como evidencia a favor de un hecho si y solo si $x$ es un indicador fiable del hecho. Por ejemplo, la caída de nivel del barómetro es evidencia de lluvia inminente ya que fiablemente indica tal estado. Y, por su puesto, estados mentales también pueden calificar como evidencia. Por ejemplo, que nos parezca ver o recordar que $p$ (en ciertas circunstancias) es generalmente un indicador fiable que $p$. Pero no todos los estados mentales son indicadores fiables de sus contenidos. Desear o esperar 
que $p$ no es un indicador fiable que $p$. Por lo tanto, si las intuiciones son o no son indicadores fiables de la verdad de sus contenidos es una pregunta abierta.

Por otra parte, como se mencionó, en la epistemología las intuiciones generalmente conciernen juicios acerca de escenarios descritos en experimentos mentales. Armamos un caso real o imaginario y preguntamos si es correcto clasificarlo como un caso de, por ejemplo, conocimiento. En base a nuestros juicios intuitivos, concluimos que es o no es un caso de conocimiento. Y si una teoría no captura la intuición, entonces la intuición cuenta como evidencia contra la teoría. De hecho, algunos piensan que las intuiciones tienen el poder para automáticamente derribar la teoría. Particularmente, en la era post-Gettier, desacuerdos con intuiciones son a menudo considerados como problemas fatales para las teorías (Shope, 1983; cf. Weatherson, 2003). Pero las intuiciones pueden ser consideradas como evidencia sin ser consideradas como evidencia que no puede ser derrotada y en lo que sigue asumiré su derrotabilidad (de hecho, paradojas, como la introducida, sugieren que eso es correcto), permitiendo así la posibilidad de que otros datos anulen epistémicamente a las intuiciones. ${ }^{12}$

(c) Por último, aquí adopto el naturalismo metodológico de los anteriormente mencionados enemigos de las intuiciones. Por supuesto no todos los naturalistas metodológicos son hostiles a las intuiciones (e.g. Goldman, 2007). De hecho, no todos los epistemólogos experimentales lo son (e.g. May et al., 2010). Estos epistemólogos experimentales "positivos" (o "constructivistas") comparten con la corriente epistemológica dominante el compromiso de emplear intuiciones como evidencia derrotable, pero asumen que sus encuestas proveen un suplemento necesario para esta práctica. ${ }^{13}$

El naturalismo metodológico, según lo entienden estos enemigos de las intuiciones, requiere por lo menos algún tipo de continuidad de resultados: una continuidad que establece que las teorías filosóficas deben ser informadas por y consistentes con la ciencia. Pero este naturalismo no parece requerir continuidad de métodos, entendida como una continuidad que establece que las teorías filosóficas deberían ser construidas solo en base a los métodos de la

12 De hecho, uno podría plausiblemente sostener que no hay razón para pensar que nuestro aparato conceptual es desarrollado para lidiar con casos bizarros (ver nota 9). En ese caso, el estatus evidencial de las intuiciones que van más allá de la práctica corriente sería socavado, ya que la práctica estaría siendo entendida de manera forzada. En efecto, existe trabajo empírico que sugiere que es menos probable que factores epistémicos irrelevantes influencien nuestras intuiciones cuando consideramos casos paradigmáticos que cuando consideramos casos difíciles que están en el límite de nuestra practica corriente (Wright, 2010). Y la investigación experimental sugiere que las intuiciones que involucran casos paradigmáticos son fiables (Liao, 2008).

13 Entonces mientras epistemólogos experimentales positivos afirman que las intuiciones pueden ayudarnos a hacer filosofía, los epistemólogos experimentales negativos lo niegan. Para más detalles sobre esta distinción y la relación entre los enfoques, ver e.g. Stich y Tobia (2016), Weinberg (2016). 
ciencia. ${ }^{14}$ Después de todo, no es claro lo que el método científico es. ${ }^{15}$ Quizá algo bien parecido al método que busca equilibrio reflexivo puede entenderse como tal (Rawls, 1951; Cummins, 1998). Pero aunque esto fuese así, uno podría de todas formas encontrar diferencias en los detalles. ${ }^{16}$ Por ejemplo, uno podría sugerir que algún elemento del método que busca equilibrio reflexivo filosófico está completamente ausente de la versión científica. Uno podría sugerir que las intuiciones (por lo menos el tipo de intuiciones aquí consideradas) son tal elemento (o uno de esos elementos). Eso entonces mostraría que la investigación científica es distinta a la filosófica aunque ambas busquen un equilibrio reflexivo. Por lo tanto, existe la posibilidad para que la metodología filosófica no sea considerada como una instancia de la metodología científica y entonces para rechazar la tesis de continuidad de métodos.

Dado lo anterior, ¿hay continuidad entre la ciencia y la filosofía? Ciertamente podría serlo en el sentido de continuidad de resultados (y para muchos lo es), pero podría no serlo en el sentido de continuidad de métodos. Pero cualquiera sea la respuesta en este último caso, una posición naturalista claramente no utiliza métodos misteriosos o supernaturales para alcanzar las explicaciones de los fenómenos investigados. ${ }^{17}$ Dado este naturalismo, podemos conceder la relevancia de los datos de las encuestas experimentales. En otras palabras, el equilibrio reflexivo buscado sería amplio (ya que involucraría distintos tipos de elementos) y no estrecho. Pero es importante notar que los datos de cualquier disciplina empírica pueden en principio ser explotados en el teorizar epistemológico (y, más ampliamente, filosófico).

\section{Resultados negativos de la epistemología experimental}

Como hemos anunciado arriba, en este artículo nos concentraremos en dos desafíos particulares (dado el trasfondo anterior). El primero consiste en capturar los resultados de la epistemología experimental negativa (I). Este

14 Leiter (2002, pp. 4-5) distingue entre continuidad de resultados y continuidad de métodos, y toma al primero como un tipo de continuidad más importante.

15 De todos modos, uno se podría preguntar si hay tal cosa como el método científico. Los que han sido influenciados por Kuhn y Feyerabend responden negativamente. Lo mismo aplica al método filosófico, pero aquí estoy ignorando, en aras del argumento, estas complicaciones.

16 Estos detalles son importantes; de lo contrario, dado que el método que busca equilibrio reflexivo es un método general de razonamiento, no podríamos distinguir entre las distintas empresas reflexivas, no solo la filosofía y la ciencia.

17 De hecho, esas explicaciones, como dice Bernard Williams (2002, p. 23), deben ser ofrecidas en términos del resto de la naturaleza (como el naturalismo metafísico requiere). Por supuesto que algunos podrían pensar que esta manera de entender el naturalismo metodológico es controvertida, pero la idea aquí es simplemente entenderlo de tal manera que, en aras del argumento, permita que los datos obtenidos de las encuestas sean relevantes al teorizar filosófico (cf. Nanay, 2015). 
trabajo empírico sugiere que las intuiciones epistémicas varían de acuerdo a factores epistémicamente irrelevantes, como factores socio-económicos. Por ejemplo, las intuiciones acerca de casos tipo Gettier parecen exhibir una variación intercultural (Weinberg et al., 2001) y las intuiciones acerca del caso Truetemp parecen ser susceptibles a efectos de orden (Swain et al., 2008). ${ }^{18}$ Los resultados de la epistemología experimental negativa introducen diferencias inter- e intrapersonales que parecen crear dudas acerca de la relevancia de las intuiciones epistémicas a la investigación epistemológica a menos que uno pueda identificar las intuiciones correctas. Los epistemólogos experimentales negativos creen que determinar qué intuiciones epistémicas pueden ser ignoradas o privilegiadas para construir teorías es un tremendo desafío (Alexander et al., 2010).

Antes de lidiar con el desafío, déjeme primero señalar algunas cosas que nos ayudarán con lo anterior. Primero, intentar responder a este desafío privilegiando las intuiciones de un grupo de individuos, porque estas son el producto de una cuidadosa reflexión o entrenamiento, no funcionará. Dejando de lado que inter- e intra-variabilidad puede también ser encontrada dentro de un grupo determinado (por ejemplo, filósofos), estas intuiciones reflexivas (aunque estuvieran exentas de variabilidad y maleabilidad) no vindicarían el objetivo de nuestra investigación: intuiciones como juicios no-inferenciales que surgen espontáneamente (ver (a) arriba). ${ }^{19}$

Segundo, las intuiciones (no reflexivas) de la gente parecen de todas formas requeridas para proveer la caracterización inicial del fenómeno a investigar (Goldman, 2007). Esto es, las intuiciones-folk tienen un rol basal para jugar en las investigaciones de fenómenos comunes. Deberíamos tomarlas seriamente ya que, si nos alejamos mucho de lo que habitualmente pensamos, podríamos terminar cambiando la materia que inicialmente nos movió a investigar y por lo tanto teorizando acerca de cosas que no serán reconocidas como importantes (ver también Lewis, 1986, p. 134; 1973, p. 88). De hecho, si una teoría no se ajusta aproximadamente a lo que comúnmente se considera como, por ejemplo, conocimiento, no habrá mucho interés en ella. Las intuiciones-folk entonces pueden ser útilmente consideradas y empleadas como una parte vital de la

18 Efectos de orden corresponden a las diferencias en las respuestas de los participantes a estudios que resultan por el orden en que los materiales del experimento son presentados. Estos efectos de orden pueden ocurrir en todo tipo de investigación. En encuestas, por ejemplo, los participantes podrían responder preguntas de forma distinta dependiendo del orden en que estas se realizan. Para más detalles al respecto, ver e.g. Shaughnessy et al. (2012, pp. 171-2).

19 Williamson (2011) propone un enfoque "elitista" (que toma en cuenta solo las intuiciones de los filósofos) que intenta lidiar con esta variabilidad. Para otras complicaciones de este enfoque, ver e.g. Kornblith (2012), Machery (2015), Weinberg et al., (2012); cf. Grundmann (2012). Más significativamente (y como veremos pronto), el enfoque "populista" (que toma en cuenta las intuiciones de la gente) tiene un rol muy importante que jugar en la filosofía. 
filosofía (cf. Aristotle N.E. 1145b1 y sig.). Y, como he sugerido, la razón por la cual este rol es importante es que deberíamos evitar posiciones filosóficas que nos resultan ajenas (Goldman, 1992, p. 272; Pritchard, 2009, p. 4). ${ }^{20}$ Por lo tanto, dado que la filosofía surge de lo común y no puede perder conexión con ello, seguramente hay un lugar para que las intuiciones-folk jueguen un rol basal en nuestra investigación epistemológica. ${ }^{21}$

Tercero, lo anterior claramente apoya el uso de encuestas acerca de nuestras intuiciones-folk para el teorizar epistemológico. Pero estas encuestas son relevantes para tal teorizar solo si son apropiadamente diseñadas y conducidas. Lamentablemente algunas dudas parecen surgir acerca de la calidad de las encuestas que deben ser consideradas. ${ }^{22}$ Por ejemplo, en Weinberg et al. (2001), la elección entre "saber realmente" y "solo cree" en los casos Gettier fue forzada en los individuos entrevistados, pero ninguna de las opciones parece correcta y el "realmente" es probable que solo introduzca ruido. ${ }^{23}$ Lo anterior es particularmente preocupante dado que las posibles explicaciones de la variaciones interculturales se basan en diferencias interculturales acerca de los aspectos de la narración del caso que son destacados y en cómo los detalles de las narraciones (no descritas con la precisión adecuada) son rellenados. Además, no es claro que los individuos de las encuestas están apropiadamente motivados a leer cuidadosamente las narraciones de los casos y prestarles suficiente atención. Nuevamente, variación intercultural en estas encuestas pueden explicarse mediante diferencias interculturales en motivación. Y, significativamente, cuando las correcciones relevantes se realizan, los resultados "negativos" no son reproducidos (por ejemplo, la clarificación de los factores pragmáticos cancela los efectos de orden en las encuestas de los casos Truetemp-Cullen, 2010).

20 Sin embargo, como una teoría que es solamente un reflejo de nuestras intuiciones y no añade nada a nuestro entendimiento del fenómeno estudiado es una teoría vacía, deberíamos intentar encontrar un término medio entre una teoría ajena y una vacía.

21 Ver también Lewis (1983, p. x), Hintikka (1999). Pero, como Austin (quien pensaba que una buena aproximación a los problemas filosóficos consistía en una examinación de lo que decimos y hacemos en la vida corriente) dice, "el lenguaje corriente no es la última palabra: en principio puede siempre ser suplementado y mejorado y reemplazado" (1970, p. 185; mi traducción). Lo mismo puede decirse de las intuiciones (y uno podría ir más lejos y sugerir, como algunos lo hacen que esas intuiciones bombeadas de situaciones artificiosas y extraordinarias no poseen la fuerza dialéctica de aquellas bombeadas de situaciones corrientes-ver notas 9 y 12).

22 De hecho, para algunos, dada la aparente falta de rigor científico involucrada en estas encuestas o (peyorativamente) "serias votaciones de opiniones" (Jackson, 1998, p. 36), no es claro que estas encuestas presentan el desafío que se supone presentan.

23 El "realmente," así también como "genuinamente," parece invitar a que uno considere el valor de verdad de la afirmación de tal manera que solo los casos más evidentes de, en este caso, conocimiento sean tomados como tal. El test del "realmente" no es fácil de pasar aún para casos legítimos de conocimiento (cf. Conee, 2014). 
Otras preocupaciones acerca de las encuestas podrían ser consideradas, ${ }^{24}$ pero no hay ninguna razón en concreto para pensar que no podemos en principio diseñar y conducir encuestas que son metodológicamente adecuadas. Por lo tanto asumiré, en aras del argumento, que no solo lo anterior es posible sino que también es probable que tales encuestas arrojen resultados negativos.

Cuarto, y no obstante lo anterior, los resultados de las encuestas deberían ser repetibles si van a ser consideradas. En otras palabras, lo resultados necesitan ser robustamente reproducidos si permitiremos que guíen nuestro teorizar. Desafortunadamente, la filosofía experimental todavía se encuentra en su infancia y no ha producido resultados robustos, en términos de repetibilidad, a pesar de algunos intentos por investigadores de lograrlo. Por ejemplo, la investigación experimental sobre los casos Gettier ha fallado en reproducir resultados negativos anteriores (e.g. Nagel, 2012; Mortensen y Nagel, 2016). De todas maneras, no hay tampoco en este caso una razón concreta para pensar que, en principio, algunos resultados podrían dar lugar al tipo de desafío que el filósofo experimental tiene en mente, y por lo tanto también ignoraré, en aras del argumento, este asunto.

Dado lo anterior, asumo que no hay razón para pensar que no podemos diseñar y conducir encuestas metodológicamente adecuadas acerca de nuestras intuiciones-folk que pueden entregar resultados negativos robustos. Entonces: ¿Cómo puede el teorizar epistemológico proceder dado el posible conflicto de intuiciones? ¿Qué intuiciones privilegiamos para construir nuestras teorías epistemológicas frente a tal conflicto? Aquí el asunto no es si las intuiciones son generalmente indicadores fiables de la verdad de sus contenidos (ver (b) arriba) y por lo tanto (buena) evidencia (Alexander y Weinberg, 2014). En cambio, el asunto es que no hay un criterio que nos ayude a decidir cuándo una intuición en particular no es un indicador fiable de la verdad. ${ }^{25}$

Siguiendo el trabajo de Edward Craig, que desea otorgarle a las intuiciones un cierto papel en el teorizar epistemológico (1990, pp. 1-3, 6-7), podemos arbitrar entre intuiciones y reducir el impacto de los posibles conflictos entre intuiciones explotando distintas fuentes de evidencia (no solo las intuiciones) y consideraciones funcionales. Esta estrategia de abrir nuevas líneas de investigación empírica debería ser, como hemos visto (ver (c) arriba), bienvenida por el naturalista metodológico.

24 Ver e.g. Sosa $(2009 ; 2012)$.

25 Cf. Cummins (1998). Cummins sugiere que cuando un instrumento arroja datos, estos califican como evidencia solo si el instrumento ha sido "calibrado," i.e. se ha mostrado independientemente que es un indicador fiable de la verdad. 
La idea no es meramente demandar más evidencia derivada de la psicología experimental, sino también extraer evidencia de otras disciplinas científicas relevantes (como ser biología, lingüística, historia, entre otras). Esta evidencia adicional puede permitir maneras alternativas de entender los resultados de las encuestas que las hace obsoletas (ver arriba) pero, más significativamente, puede proveer un mayor capital de evidencia que disminuye la importancia de los posibles conflictos. Las intuiciones no son los únicos ítems de evidencia disponibles y la evidencia total en algunos casos puede favorecer una posición $u$ otra a pesar de las intuiciones en conflicto. Por lo tanto, considerar una selección más amplia de evidencia puede ayudar a reducir el impacto de las intuiciones discrepantes en el teorizar epistemológico. ${ }^{26}$

Por otra parte, deberíamos prestarle atención a los roles que los fenómenos investigados (por ejemplo, el conocimiento) juegan en nuestras vidas ya que nos pueden ayudar aún más a arbitrar entre intuiciones. Craig (1990, p. 11) aspira a dilucidar el rol social del conocimiento considerando hechos acerca de nuestro entorno físico, nuestra organización social y nuestro carácter psicológico y biológico (particularmente, acerca de los objetivos e intereses que típicamente poseemos).

Para nuestros propósitos, los detalles de la posición de Craig no son relevantes, pero la idea es que podemos apreciar en ese contexto que hay una necesidad general que el fenómeno a ser explicado, en este caso el conocimiento, satisface (1990, p. 10). ${ }^{27}$ Y lo que importa es que esta función del conocimiento sirve para determinar qué intuiciones pueden ser consideradas incorrectas y por lo tanto ser explicadas por medio de una teoría de error (sin esta explicación ser ad hoc). Esto es, una ventaja importante, no comúnmente explotada, de tales explicaciones prácticas es que las consideraciones funcionales pueden ser invocadas para evaluar la importancia de las distintas intuiciones. Como Ruth Weintraub dice,

Las consideraciones funcionales sirven también para determinar si un contra-ejemplo podría ser rechazado como una instancia de ‘uso secundario' de un término. Tal rechazo no puede ser siempre descartado como ad hoc [. ... Por lo tanto] consideraciones

26 Esta mayor selección de evidencia podría proveernos con buenas razones independientes para pensar que algunas intuiciones son incorrectas en algunos casos de divergencia. Considere por ejemplo el caso de gente con intuiciones racistas. En estos casos, la divergencia de intuiciones no es problemática para la construcción de teorías, dadas las razones independientes. Además, aunque no tengamos una teoría de error para estas intuiciones, la opción más razonable es tomar al grupo con esas intuiciones (i.e. los racistas) como jueces no fiables del tema considerado, en vez de adoptar un escepticismo general acerca de las intuiciones (éticas).

27 Para más detalles sobre la posición de Craig y la aproximación detrás de ella, ver e.g. Kappel (2010), De Brasi (2015a). 
funcionales podrían ser invocadas para evaluar la importancia de (contra-) ejemplos para un análisis (1991, p. 159; mi traducción).

De hecho, algunos naturalistas ya ejercen prácticas similares a las descritas arriba. Por ejemplo, Hilary Kornblith (2002) explota evidencia de la etología cognitiva y, en particular, el rol que el conocimiento juega en esa disciplina para apoyar la posición que el conocimiento es creencia verdadera fiablemente formada. Por lo tanto Kornblith puede explotar el rol del conocimiento en la etología cognitiva para afirmar que, por ejemplo, los veredictos negativos en los casos Truetemp son incorrectos. De manera similar, uno puede sacar provecho de la función social del conocimiento para descartar intuiciones (e.g. De Brasi, 2015a). Entonces estas nuevas líneas de investigación nos ayudan a resolver qué intuiciones pueden ser consideradas como "ruido." Y, aunque no tienen por qué ser incompatibles, si lo fuesen (y gozaran del mismo apoyo evidencial), resolveríamos la inconsistencia decidiendo qué debe ser descartado en base a una evaluación de los beneficios y costos teóricos.

Ahora bien, por supuesto que la divergencia per se no implica que algunas intuiciones sean incorrectas. Si el objetivo de la investigación filosófica fuese el concepto de un individuo (i.e. el contenido del concepto conocimiento de un individuo dado), entonces la divergencia entre individuos no tiene por qué sugerir que alguna intuición es incorrecta. ${ }^{28}$ Pero eso representaría una seriamente errónea caracterización de muchos proyectos filosóficos, ya que estamos interesados no meramente en conceptos sino en las cosas mismas. Por ejemplo, no estamos meramente interesados en el concepto conocimiento sino también en el conocimiento mismo (i.e. la relación epistémica objetiva). Es por esto que necesitamos, siguiendo a Craig, tomar en consideración, por ejemplo, consideraciones funcionales para arbitrar entre intuiciones encontradas. Y porque la filosofía no tiene como objetivo meramente entender conceptos en las cabezas de distintos individuos, entonces nos enfrentamos a otro desafío.

\section{Desajustes entre conceptos y categorías epistémicas}

El segundo desafío que consideraremos aquí concierne la posibilidad de un desajuste entre nuestros conceptos de los fenómenos a ser investigados y los fenómenos mismos (II). Este desafío debe ser tomado en serio si se supone

28 Eso, sin embargo, no descarta otras posibilidades de error, como errores por performance. Después de todo, cuando uno lee descripciones de casos (especialmente estrambóticos), uno puede no darse cuenta de ciertos detalles importantes y de ese modo formar intuiciones incorrectas. 
que las intuiciones son evidencia a favor de una realidad externa que podemos razonablemente esperar captarla de manera fallida. Después de todo, uno podría preguntarse cómo nuestras mentes pueden discernir esos contenidos. ${ }^{29}$ Nuestras intuiciones, algunos podrían pensar, pueden a lo sumo revelar nuestros conceptos pero los conceptos no son, como hemos visto, (siempre) los objetos del teorizar epistemológico. Esta potencial brecha entre conceptos y fenómenos a investigar parece sugerir que las intuiciones no deberían jugar ningún rol en la investigación epistemológica.

Sin embargo, note que si la mera posibilidad de error estuviera siendo aprovechada en este caso para crear el desafío, podríamos descartarlo simplemente sobre la base que asume estándares extremadamente altos para las creencias razonables. ${ }^{30}$ De todos modos, esta posibilidad podría parecer significativa si pensamos acerca del fenómeno a ser investigado como una clase natural (como ser el agua y el oro), dado que este sería un fenómeno que existiría independientemente de nuestros estados mentales y prácticas sociales (Kornblith, 2006).

Pero aunque concedamos que hay una posibilidad significativa de que nuestros conceptos no están adecuadamente en sintonía con rasgos de las clases naturales, podemos sugerir, siguiendo a Craig, que algunas categorías epistémicas, como el conocimiento, son clases sociales: aproximadamente, categorías que los seres humanos imponen en el mundo en respuesta a ciertas necesidades e intereses. Más específicamente, clases sociales son categorías causalmente significativas (como las clases naturales lo son) que nosotros creamos en las transacciones que definen nuestra vida social (Mallon, 2016). Entonces, estas categorías (que por ejemplo prácticas sociales podrían producir) refieren a aspectos del mundo y su relevancia e importancia las hacen candidatas para la inclusión en nuestras mejores y más exitosas teorías. En el caso del conocimiento, como dice Craig, este es "algo que nosotros delineamos por medio de un concepto que creamos en respuesta a ciertas necesidades" (1990, p. 3). ${ }^{31}$ Asumiendo que esto es así, nuestro concepto de conocimiento juega un

29 Un perseverante problema que se remonta por lo menos a la teoría de las formas de Platón.

30 Para más detalles acerca de posibilidades de error relevantes, ver e.g. De Brasi (2014).

31 Nada de lo anterior sugiere que el conocimiento no es un fenómeno natural o es incompatible con el naturalismo metodológico aquí considerado (ver e.g. Haslanger, 2012; Mallon, 2016), pero simplemente una clase social cuya existencia depende en parte de ciertos factores humanos y que podría en principio cambiar. Tampoco esto significa que el conocimiento no es una relación epistémica objetiva. De hecho, una epistemología Craiguiana no tiene por qué ser susceptible a los (a menudo percibidos) excesos del "programa fuerte" de la sociología del conocimiento (e.g. Bloor, 1991) y otras epistemologías sociales "revisionistas"-según la clasificación de Goldman (2010). Para más detalles, ver De Brasi (2015b). 
rol crucial en determinar lo que el conocimiento es, ${ }^{32}$ lo que nos permite darle sentido al hecho de que nuestras intuiciones pueden revelar su naturaleza.

Ahora bien, por supuesto que este enfoque no garantiza la exactitud de nuestras intuiciones, como Kornblith (2006, p. 21) correctamente señala. Pero esto no debe ser visto como un problema sino una ventaja, ya que nos permite capturar la posibilidad de ignorancia y error acerca de la clase. Y, lo que es más importante, Kornblith falla en proveer cualquier tipo de razones concretas para sugerir que, en el caso de clases sociales comunes y "extremadamente prácticas” (Craig, 1990, p. 4), podríamos no estar más acertados en capturar aspectos de esas clases que de las clases naturales (Kornblith, 2006, p. 21; 2007, p. 37). Kornblith simplemente no provee razones y, como he sugerido, si solo es introducida la mera posibilidad de que estos conceptos podrían contener errores y omisiones, entonces podemos razonablemente descartar el desafío. ${ }^{33}$

Kornblith podría estar consciente de esto y por eso quejarse de que Craig no nos ofrece "una razón para creer que la categoría de conocimiento es una construcción social en vez de una clase natural" (2011, p. 49; mi traducción). Pero Craig (1990, p. 1) está solo desarrollando una aproximación alternativa dados los fracasos de la corriente epistemológica (post-Gettier) dominante y esta aproximación, como cualquier otra, debe ser juzgada en última instancia por sus frutos teóricos. Esto es, la afirmación de Craig de que el conocimiento es una clase social debe ser tomada como una plausible presunción metodológica (tal

32 Ver e.g. De Brasi (2015a).

33 Para ser justo, Kornblith (2015) aclara que él no cree que esta es una mera posibilidad de error (aun tratándose de clases sociales) pero, lamentablemente, en una nota al pie dice que ha tratado esta posibilidad relevante de error con especial atención a las clases sociales en Kornblith (2006). Y, a continuación, solo afirma que "es muy común que nuestros conceptos fracasen en capturar completamente o correctamente todos los aspectos esenciales de las cosas de las cuales son conceptos" (2015, p. 152; mi traducción y énfasis) y, en una nota al pie, sugiere que en Kornblith (2014) discute errores y omisiones que se supone son pertinentes. Desafortunadamente, eso no es así. Es claro que Kornblith (2014, p. 208) tiene en mente los frecuentes errores y omisiones de métodos que se pueden llevar a cabo completamente desde el sillón (esto es, que ignoran descubrimientos empíricos). Pero ese tipo de metodología no es la que el naturalista metodológico (que nos concierne) asume y, significativamente, no es el único tipo de metodología que puede explotar el uso evidencial de las intuiciones (como hemos visto aquí). Lo importante es advertir que Kornblith (2014) argumenta en contra de un tipo de metodología del sillón y no contra todo uso de las intuiciones como evidencia per se. Kornblith en ese artículo también afirma cosas como: "Cuando teorías filosóficas en la epistemología se construyen desde el sillón, corren un serio riesgo de estar divorciadas de los fenómenos que intentan iluminar. La única manera de asegurar que no construimos intricados castillos en el aire, no conectados a los fenómenos reales que motivaron nuestro trabajo inicialmente, es basar nuestro trabajo en el mejor entendimiento empírico de esos fenómenos" (2014, p. 207; mi traducción). Pero aunque estoy de acuerdo con su primera aseveración (dado que, por ejemplo, trabajo empírico o basado en resultados empíricos es necesario para arbitrar entre intuiciones, como hemos visto-§3), no es claro que, como parece sugerir la segunda aseveración (lo cual es desde ya la intención de Kornblith), no haya un rol basal para las intuiciones (tal como hemos visto anteriormente). 
como lo es aquíi ${ }^{34}$ ) y la mejor manera de avanzar es asumir que tal presunción es correcta y ver donde eso nos lleva (Craig, 1990, p. 4). ${ }^{35}$ Mientras no haya razones para pensar que la posibilidad de error anteriormente mencionada es más que una mera posibilidad, esta presunción metodológica desarma el desafío en cuestión (ya que tal posibilidad por sí sola no constituye una razón concreta para considerar que un error es probable) y por lo tanto las intuiciones pueden gozar el rol de evidencia en el teorizar epistemológico.

\section{Conclusión}

Para concluir, la epistemología Craiguiana puede ayudar al naturalista metodológico a reivindicar el familiar uso de las intuiciones como evidencia derrotable, sin tener que asumir fuertes compromisos acerca de nuestra arquitectura cognitiva, como algunos lo hacen (e.g. Shieber, 2012; Spicer, 2007). Entonces, el revuelo que se ha generado recientemente en torno a la ilegitimidad de este uso de las intuiciones parece precipitado, por lo menos para tal naturalista. Además, como hemos visto, esta aproximación Craiguiana puede otorgarle un útil rol epistémico a la epistemología experimental (positiva) en la construcción de teorías (cf. Kornblith, 2015; Nanay, 2015). Por supuesto esta podría no ser la aproximación correcta al conocimiento y otros fenómenos epistémicos (y desde ya que aquí no he intentado motivarla de manera independiente). Pero la manera más efectiva de adjudicar entre aproximaciones es desarrollarlas en detalle y explorar sus fortalezas y debilidades. Y uno explota intuiciones poniendo en práctica esta aproximación Craiguiana (y otras tantas también), por lo tanto independientemente del eventual éxito de alguna aproximación particular que no le otorgue un rol evidencial a las intuiciones, aún existe un rol para que las intuiciones jueguen en el teorizar epistemológico dada esta adjudicación entre aproximaciones.

\section{Bibliografía}

ALEXANDER, J. "Experimental Philosophy.” Cambridge: Polity, 2012.

ALEXANDER, J., MALLON, R., WEINBERG, J. "Accentuate the Negative." Review of Philosophy and Psychology, 1, pp. 297-314, 2010.

ALEXANDER, J., WEINBERG, J. "Analytic Epistemology and Experimental

34 Para una motivación independiente a favor de la tesis que el conocimiento es una clase social, ver De Brasi (2017).

35 Para otros frutos teóricos de la aproximación (más allá de legitimar el uso de las intuiciones como evidencia en el teorizar epistemológico), ver De Brasi (2015a). 
Philosophy." Philosophy Compass, 2/1, pp. 56-80, 2007.

. "The 'Unreliability' of Epistemic Intuitions." (pp. 128-145). In: E. Machery, E. O’Neill (eds.). Current Controversies in Experimental Philosophy. London: Routledge, 2014.

ARISTOTLE. “A New Aristotle Reader.” Ed. J. L. Ackrill. Oxford: OUP, 1987.

AUSTIN, J. L. "Philosophical Papers." Oxford: Clarendon Press, 1970.

BAUMANN, P. “Epistemic Closure.” (pp. 597-608). In: S. Bernecker, D. Pritchard (eds.). The Routledge Companion to Epistemology. London: Routledge, 2011.

BEALER, G. "Intuition and the Autonomy of Philosophy." (pp. 201-240). In: W. Ramsey, M. De Paul (Eds.). Rethinking Intuition: The Psychology of Intuition and its Role in Philosophical Inquiry. Maryland: Rowan \& Littlefield, 1998.

BLOOR, D. "Knowledge and Social Imagery". $2^{\text {nd }}$ ed. Chicago: Chicago University Press, 1991.

BOOTH, A., ROWBOTTOM, D. "Intuitions.” Oxford: OUP, 2014.

CAPPELEN, H. "Philosophy Without Intuitions." Oxford: OUP, 2012.

CHISHOLM, R. "The Foundations of Knowing." Minneapolis: University of Minnesota Press, 1982.

CHUDNOFF, E. "Intuition." Oxford: OUP, 2013.

COHEN, S. "How to be a Fallibilist." Philosophical Perspectives, 2, pp. 91-123, 1988. CONEE, E. “Is Knowledge Contextual?” (pp. 60-68). In: M. Steup, J. Turri, E. Sosa (eds.). Contemporary Debates in Epistemology. Oxford: Blackwell, 2014.

CRAIG, E. "Knowledge and the State of Nature." Oxford: Clarendon, 1990.

CULlEN, S. "Survey-Driven Romanticism." Review of Philosophy and Psychology, 1, pp. 275-96, 2010.

CUMMINS, R. "Reflections on reflective equilibrium." (pp. 113-128). In: W. Ramsey, M. De Paul (eds.). Rethinking Intuition: The Psychology of Intuition and its Role in Philosophical Inquiry. Maryland: Rowan \& Littlefield, 1998.

DANIELS, N. "Wide Reflective Equilibrium and Theory acceptance in Ethics." Journal of Philosophy, 76, pp. 256-82, 1979.

DE BRASI, L. "Closure Intuitions and Restriction." Praxis Filosófica - Nueva Serie, 38, pp. 225-248, 2014.

. "Testimony and Value in the Theory of Knowledge." Ideas y Valores, Vol. 64, Nr. 159, pp. 87-107, 2015a.

."Reliability and Social Knowledge-Relevant Responsibility." Transformaçao,

Vol. 38, Nr. 1, pp. 187-212, 2015 b.

"Knowledge as a Social Kind." Filosofía Unisinos, Vol. 18, Nr. 3, pp.130$139,2017$.

DENNETT, D. "The Milk of Human Intentionality.” Behavioral and Brain Sciences, 3, pp. 429-430, 1980.

. "Fast Thinking." (pp. 324-337). In: D. Dennett. The Intentional Stance.

Cambridge: MIT Press, 1987.

. "Intuition Pumps and Other Tools for Thinking." NY: W.W. Norton \& Co, 
2013.

DENNETT, D., HOFSTADER, D. “The Mind's I.” London: Penguin, 1982.

DESCARTES, R. "The Philosophical Writings of Descartes." Vols. I \& II (Trans. Cottingham, Stoothoff \& Murdoch). Cambridge: CUP, 1984-5.

DEUTSCH, M. "The Myth of the Intuitive." Cambridge: MIT Press, 2015. DRETSKE, F. "Fred Dretske.” (pp. 130-134). In: J. Dancy, E. Sosa, M. Steup (eds.). A Companion to Epistemology ( $2^{\text {nd }}$ edition). Oxford: Blackwell, 2010.

GETTIER, E. “Is Justified True Belief Knowledge?” Analysis, 23, pp. 121-3, 1963. GOLDMAN, A. "Epistemic Folkways and Scientific Epistemology." Philosophical Issues, 3, pp. 271-285, 1992.

."Philosophical Intuitions: Their Target, Their Source, and Their Epistemic Status." Grazer Philosophische Studien, 74, pp. 1-26, 2007.

. "Why Social Epistemology Is Real Epistemology." (pp. 1-28). In: A. Haddock, A. Millar, D. Pritchard (eds.). Social Epistemology. Oxford: OUP, 2010. GRECO, J. "Putting Skeptics in Their Place." Cambridge: CUP, 2000.

GRUNDMANN, T. "Some hope for intuitions: A reply to Weinberg". (pp. 199228). In: J. Horvath, T. Grundmann (eds.). Experimental Philosophy and Its Critics. London: Routledge, 2012.

HASLANGER, S. "Resisting Reality.” Oxford: OUP, 2012. HAWTHORNE, J. "Knowledge and Lotteries." Oxford: OUP, 2004.

"Is Knowledge Closed under Known Entailment?" (pp. 40-55). In: M. Steup, J. Turri, E. Sosa (eds.). Contemporary Debates in Epistemology. Oxford: Blackwell, 2014.

HINTIKKA, J. “The Emperor's New Intuitions.” Journal of Philosophy, 96, pp. 12747, 1999.

JACKSON, F. "From Metaphysics to Ethics." Oxford: Clarendon, 1998.

KAPPEL, K. "On Saying that Someone Knows: Themes from Craig". (pp. 69-88). In:

A. Haddock, A. Millar, D. Pritchard (eds.). Social Epistemology. Oxford: OUP, 2010. KELLY, T. "Evidence: Fundamental Concepts and the Phenomenal Conception." Philosophy Compass, 3, pp. 933-955, 2008.

KORNBLITH, H. "Knowledge and its Place in Nature." Oxford: OUP, 2002.

. "Appeals to Intuition and the Ambitions of Epistemology." (pp. 10-25). In: S.

Hetherington (ed.). Epistemology Futures. Oxford: OUP, 2006.

. "Naturalism and Intuitions." Grazer Philosophische Studien, 74, pp. 27-49, 2007. 38-52, 2011.

"Why Should We Care About the Concept of Knowledge?" Episteme, 8, pp.

. "On Reflection.” Oxford: OUP, 2012.

"Is There Room For Armchair Theorizing in Epistemology?" (pp. 195-216).

In: M. Haug (ed.). Philosophical Methodology. London: Routledge, 2014.

. "Naturalistic Defenses of Intuition." (pp. 151-168). In: E. Fischer, J. Collins (eds.). Experimental Philosophy, Rationalism, and Naturalism. London: Routledge, 2015 . 
KRIPKE, S. "Naming and Necessity." Oxford: Blackwell, 1980.

LEHRER, K. "Theory of Knowledge." Boulder: Westview Press, 1990.

LEITER, B. "Nietzsche on Morality." London: Routledge, 2002.

LEWIS, D. K. "Counterfactuals.” Oxford: Blackwell, 1973.

. "Philosophical Papers." Vol. 1. Oxford: OUP, 1983.

."On The Plurality of Worlds." Oxford: Blackwell, 1986.

LIAO, M. “A Defense of Intuitions.” Philosophical Studies, 140, pp. 247-62, 2008.

MACHERY, E. “The Illusion of Expertise.” (pp. 188-203). In: E. Fischer, J. Collins (eds.). Experimental Philosophy, Rationalism, and Naturalism. London: Routledge, 2015.

MALLON, R. "The Construction of Human Kinds.” Oxford: OUP, 2016.

MAY, J., SINNOTT-ARMSTRONG, W., HULL, J., ZIMMERMAN, A. "Practical Interests, Relevant Alternatives, and Knowledge Attributions: An Emperical Study." Review of Philosophy and Psychology, 1, pp. 265-73, 2010.

MORTENSEN, K., NAGEL, J. "Armchair-Friendly Experimental Philosophy." (pp. 53-70). In: J. Sytsma, W. Buckwalter (eds.). A Companion to Experimental Philosophy. Oxford: Blackwell, 2016.

NAGEL, J. "Intuitions and Experiments: A Defense of the Case method in Epistemology." Philosophy and Phenomenological Research, 85, pp. 495-527, 2012. NANAY, B. "Experimental Philosophy and Naturalism." (pp. 222-239). In: E. Fischer, J. Collins (eds.). Experimental Philosophy, Rationalism, and Naturalism. London: Routledge, 2015.

NOZICK, R. "Philosophical Explanations.” MA: Harvard University Press, 1981. PLATO. "Complete Works". Ed. J. M. Cooper. Indianapolis: Hackett, 1997.

POLLOCK, J. "Knowledge and Justification.” NJ: Princeton University Press, 1974. PRITCHARD, D. "Knowledge.” Hampshire: Palgrave, 2009.

RAWLS, J. “Outline of a Decision Procedure.” Philosophical Review, 60, pp. 177-97, 1951.

"The Independence of Moral Theory." Proceedings and Addresses of the American Philosophical Association, 48, pp. 5-22, 1975.

SHAUGHNESSY, J., ZECHMEISTER, E., ZECHMEISTER, J. "Research Methods in Psychology." New York: McGraw-Hill, 2012.

SHIEBER, J. "A Partial Defense of Intuition on Naturalistic Grounds." Syntheses, 187, pp. 321-41, 2012.

SHOPE, R. "The Analysis of Knowing." New Jersey: Princeton University Press, 1983.

SOSA, E. "Knowledge in Perspective." Cambridge: CUP, 1991.

"A Defense of the Use of Intuitions in Philosophy." (pp. 101-112). In: D.

Murphy, M. Bishop (eds.). Stich and His Critics. Oxford: Blackwell, 2009.

. "Intuitions and Meaning Divergence." (pp. 137-144). In: J. Horvath, T. Grundmann (eds.). Experimental Philosophy and Its Critics. London: Routledge, 2012. 
SPICER, F. "Knowledge and the Heuristics of Folk Psychology." (pp. 354-383). In: V. Hendricks, D. Pritchard (eds.). New waves in Epistemology. Hampshire: Palgrave, 2007.

STICH, S., TOBIA, K. "Experimental Philosophy and the Philosophical Tradition". (pp. 5-21). In: J. Sytsma, W. Buckwalter (eds.). A Companion to Experimental Philosophy. Oxford: Blackwell, 2016.

SWAIN, S., ALEXANDER, J., WEINBERG, J. "The Instability of Philosophical Intuitions: Running Hot and Cold on Truetemp." Philosophy and Phenomenological Research, 76, pp. 138-55, 2008.

WEATHERSON, B. "What Good are Counterexamples?" Philosophical Studies, 115, pp. 1-31, 2003.

WEINBERG, J. "The Promise of Experimental Philosophy and the Inference to Signal.” (pp. 193-208). In: J. Beebe (ed.). Advances in Experimental Epistemology. London: Bloomsbury, 2014.

. "Going Positive by Going negative: On Keeping X-Phi Relevant and dangerous." (pp. 71-86). In: J. Sytsma, W. Buckwalter (eds.). A Companion to Experimental Philosophy. Oxford: Blackwell, 2016.

WEINBERG, J, GONNERMAN, C., BUCKNER, C., ALEXANDER, J. "Are Philosophers Expert Intuiters?" (pp. 49-74). In: J. Horvath, T. Grundmann (eds.). Experimental Philosophy and Its Critics. London: Routledge, 2012.

WEINBERG, J., NICHOLS, S., STICH, S. "Normativity and Epistemic Intuitions." Philosophical Topics, 29, pp. 429-60, 2001.

WEINTRAUB, R. “Epistemology without Knowledge?” Ratio, 4, pp. 157-69, 1991. WILLIAMS, B. "Truth and Truthfulness.” NJ: Princeton University Press, 2002. WILLIAMSON, T. "Philosophical Expertise and The Burden of Proof." Metaphilosophy, 42, pp. 215-29, 2011.

WRIGHT, J.C. "On intuitional stability: The clear, the strong, and the paradigmatic." Cognition, 115, pp. 491-503, 2010. 